\begin{abstract}
"Mircea cel Batran" Naval Academy Scientific Bulletin, Volume XX - 2017 - Issue 1 The journal is indexed in: PROQUEST / DOAJ / Crossref / EBSCOhost / INDEX COPERNICUS / DRJI / OAJI I JOURNAL INDEX I I2OR / SCIENCE LIBRARY INDEX / Google Scholar / Academic Keys/ ROAD Open Access I Academic Resources / Scientific Indexing Services / SCIPIO / JIFACTOR
\end{abstract}

\title{
PSYCHO - ENTRE ANGOISSE ET PLAISIR
}

\author{
Corina SANDIUC ${ }^{1}$ \\ ${ }^{1}$ Assistant Mircea cel Batran Naval Academy, ema_sandiuc81@yahoo.co.uk
}

\begin{abstract}
Dans cet article, nous examinerons Psycho, un thriller d'un suspense farouche qui a été à la fois très populaire et très bien reçu par les critiques. Dans ce film le plaisir dramatique mène le spectateur à établir des liens spectatoriels avec un tueur de telle manière qu'il se trouve la victime d'une identification peu confortable avec celui-ci, identification qui soulève des questions sur le mal en chacun de nous, mais qui permet aussi une maîtrise partielle de nos craintes de ce mal par la libération cathartique. Psycho est l'expression d'une oeuvre qui défie les règles et perturbe le spectateur. L'étrange mélange d'enthousiasmer et de plaire au public, d'une part, et de le perturber et le troubler, d'autre part (parfois dans le même cadre) a offert à Hitchcock un statut presque unique de cinéaste qui a toujours été rentable et qui est également considéré comme un génie et un innovateur sans égal. Pour obtenir la meilleure canalisation de l'émotion et les meilleures réactions du public lors du spectacle, Hitchcock utilise les ressources de son imagination qu'il met au service de la mise en scène et du montage. Ainsi le plaisir est acquis à travers les fortes émotions véhiculées par le medium cinématographique, l'identification avec les personnages, la structure du film, c'est-à-dire le montage des scènes, et l'intrigue taquine.
\end{abstract}

Mots-clés: identification cinématographique, plaisir, suspense, montage cinématographique.

Alfred Hitchcock est considéré, à juste titre, le créateur du "thriller de suspense”. Tous ses films pourraient être considérés des thrillers dans un certain sens, car le public s'enthousiasme sans cesse pour les surprises innovatrices de ses films. Mais l'oeuvre qui a pris par surprise le public et la critique le plus est sans doute Psycho (1960). Grace à Psycho, Hitchcock est crédité d'avoir inventé le slasher movie psychologique moderne. $^{1}$ Le slasher movie habituellement considéré comme une sous-genre de l'horreur, est peut-être la forme la plus populaire du film d'horreur d'aujourd'hui, en ce qu'il remplace le monstre d'origine surnaturelle avec un monstre qui est d'autant plus effrayant parce qu'il est pleinement humain.

Au moment de la parution de Psycho, beaucoup de critiques l'ont d'abord vu comme une «tache sur une carrière honorable", mais seulement pour changer d'avis plus tard, quand ils se sont rendus compte de sa grandeur (par exemple Bosley Crowther, un critique de New York Times). ${ }^{2}$ Le public n'a pas souffert de cette ambivalence, car Psycho a gagné plus d'argent que n'importe quel autre film d'Hitchcock.

J. Hoberman considère que «Psycho devrait être vu au moins trois fois par un amateur de cinéma, la première fois pour la terreur de l'expérience [...], la deuxième fois pour la comédie macabre inhérente à la conception du film, et la troisième pour l'ensemble des significations et des symboles cachés sous la surface $[. .].)^{3}$ C'est à nous maintenant de regarder et analyser ce qui fait que Psycho soit le plus terrifiant et le plus troublant film hitchcokien.

\footnotetext{
${ }^{1}$ John C. Lyden, 2003, p.226.

${ }^{2}$ Cité dans David Sterritt, 1993, p. 100.

${ }^{3}$ J. Hoberman, 2007, p. 210 (ma traduction).
}

Le film commence par une vue aérienne de Phoenix, Arizona, qui apporte le spectateur par la fenêtre d'un hôtel dans une pièce où Sam et Marion sont presque nus, clairement juste après avoir eu des rapports sexuels. Marion dit à Sam qu'elle ne veut plus se réunir en secret, qu'elle veut se marier. Sam, cependant, insiste qu'ils ne peuvent pas se marier avant d'avoir plus d'argent. Piégée par le besoin de respectabilité, Marion veut désespérément l'argent dont elle a besoin pour échapper à sa situation difficile.

Quand Marion s'offre à nos regards insinuant l'acte sexuel, notre attitude est à la fois de convoitise et de mépris car nous sommes séduits par la nudité, mais notre conscience de spectateur moral et respectable ne peut pas estimer une femme qui accepte faire l'amour dans une chambre d'hôtel borgne, en plein après-midi. La solution au problème de Marion est offerte quand elle retourne au travail et son patron lui demande de mettre $40000 \$$ à la banque. Mais Marion part à la maison avec l'argent et le met sur son lit. Elle se trouve actuellement en sousvêtements noirs, par rapport au blanc de la première scène, comme pour indiquer un changement dans sa nature. Puis, on la trouve dans sa voiture, en s'imaginant la surprise de Sam. Malheureusement, son patron la voit, mais elle l'évite nerveusement. Au cours de son voyage pour aller voir Sam, nous sommes donnés de nombreux gros plans de son visage inquiet. II y a peu de parler, mais la musique accroît la tension. Dans la même partie du film, elle arrête la voiture pour dormir pour la nuit, quand un policier motocycliste la réveille pour la questionner. II est méfiant, et même si elle parvient à s'enfuir de lui, il la suit dans sa ville. Comme elle pousse dans l'obscurité et la pluie, elle s'imagine les soupçons du policier et de son patron, de sorte qu'il semble 
que son plan mal conçu est voué à l'échec. À ce moment-là, elle arrive au Motel de Norman Bates.

L'inquiétude de la menace posée par le motard semble être partiellement soulagée quand Marion descend au motel. On se sent plus à l'aise, après une telle chasse on a besoin de se reposer. Mais l'étrangeté des lieux et de la hôte provoquent en nous une sourde angoisse. On doit noter ici que depuis un certain temps dans le film, nous nous sommes totalement identifiés avec Marion, car l'action et la caméra l'ont suivie sans cesse. Sans qu'on le sache, nous nous sommes faits les complices de son vol, et en tant que complices, nous avons senti son anxiété.

A ce sujet, Douchet croit que parce que nous ne pouvons pas éprouver du respect à coté du comportement de Marion, nous lui attribuons les tendances que nous refoulons, dans ce cas, notre désir de vol. Ainsi son vol devient notre vol, son argent le nôtre. ${ }^{4}$ C'est intéressant de remarquer ici comme Hitchcock fait que notre attention se concentre sur d'autres choses que ceux qui l'intéressent vraiment. Nous sommes les victimes de son piège, nous nous impliquons dans le vol et l'inquiétude s'empare de nous au moment ou Marion est suivie par le motard après le vol comme si celui-ci incarnait notre sentiment de culpabilité. $^{5}$

Comme Hitchcock lui-même l'avoue dans son entretien avec Truffaut, la première partie de l'histoire est « un hareng rouge », c'est-à-dire un truc destiné à détourner l'attention du spectateur pour que le meurtre soit intensifié, afin qu'il constitue une surprise totale car il faut: «diriger complètement les pensées du spectateur. Plus nous donnons de détails sur le voyage en automobile de la fille, plus vous êtes absorbé par sa fugue et c'est pour cela que nous donnons autant d'importance au policier motocycliste aux lunettes noires et au changement d'automobile [...] On tourne et on retourne le public, on le maintient aussi loin que possible de ce qui va se dérouler.» ${ }^{6}$

Au motel, nous sommes introduits à Norman Bates, qui est sympathique et utile à notre héroine. Il l'invite à l'accompagner pour dîner dans sa maison près du motel, avec sa mère. Pendant qu'elle défait ses valises dans la chambre et cache le vol de l'argent dans un journal, on entend un argument entre Norman et sa mère, qui trouve l'idée de son invitation à Marion "dégoûtante". Après ça, Marion et Norman partagent un sandwich pour le dîner dans le bureau de Norman, qui est rempli d'oiseaux de

\footnotetext{
${ }^{4}$ Jean Douchet, 1999, p. 140.

${ }^{5}$ John Lyden, op.cit., p. 234.

${ }^{6}$ François Truffaut, 1983, pp. 230-231.
}

proie empaillés, il s'avère que son hobby est la taxidermie, et qu'il préfère les oiseaux, car ils semblent tellement «passives». Ils ne sont pas aussi passive, toutefois, lorsque nous voyons un oiseau particulièrement menaçant au-dessus de la tête de Norman. Norman pourrait être sa proie, ou un autre prédateur comme lui, pourvu que l'on considère Norman comme la victime ou pas. En fait, que Norman soit une victime ou un prédateur n'est pas vraiment clair à ce point et d'une certaine façon, ce sera le cas jusqu'à la fin du film et au delà. Au cours de leur conversation Norman dit à Marion: «nous sommes tous pris dans nos propres pièges et aucun de nous n'en peut jamais sortir.» Leur conversation se termine par Marion lui disant son vrai nom, et qu'elle a décidé de retourner à Phoenix le jour suivant «pour [se] tirer de [son] piège». On comprend alors qu'elle a décidé de retourner l'argent, «notre argent».

Dans la scène suivante, Marion est assise à un bureau dans sa chambre dans le Motel de Bates. Sur une feuille de papier brouillon, elle calcule combien elle conserve encore de l'argent qu'elle avait volé. Elle déchire le papier, va à la salle de bains, jette les morceaux dans la toilette, et ensuite avec désinvolture enlève son peignoir. Elle est maintenant nue, mais on ne voit que ses épaules et ses jambes. Quand elle entre dans la baignoire de sa douche, la caméra est placée à l'extérieur du rideau de douche. Une fois qu'elle est dans la douche et ensuite pour la plupart de la scène, la caméra est aussi à l'intérieur, en regardant d'abord son visage pendant qu'elle jouit de l'écoulement de l'eau sur elle. L'écoulement de l'eau descendant de la douche augmente le sentiment de confort et d'abandon qu'elle ressent. En nous permettant d'observer Marion se déshabiller à travers un trou dans la cloison, Hitchcock nous rappelle notre propre voyeurisme et, en ce sens, démontre que Norman (et le spectateur aussi) a plus de désirs, qu'il veut admettre. En parlant du voyeurisme spectatoriel chez Hitchcock, Esquenazi l'explique de la manière suivante: «il lui permet de profiter du tableau envoûtant de la star, tout en affirmant que la fascination spectatorielle est celle du héros. Le spectateur peut en jouir, et tenir à distance cette jouissance dont il suppose qu'elle est éprouvée par le héros. ${ }^{7}$

Duchet observe qu'au moment ou Marion décide de retourner nos dollars, nos expectations sont déçues. Dès lors, elle ne nous intéresse plus. «Nous la sacrifions à notre appétit de voyeur. ${ }^{8}$ Mais il semble que Norman, comme nous, est un voyeur et il y a transfert - Norman devient notre héros. Marion devient maintenant ce qu'elle l'avait

\footnotetext{
${ }^{7}$ Pierre Esquenazi, 2001, p. 126.

${ }^{8}$ Jean Douchet, op. cit., p. 143.
} 
toujours été : un objet de convoitise pour notre instinct sexuel. Nous redevenons le voyeur du début du film quand nous avions pénétré dans la chambre par les volets clos. Douchet note que Marion en se déshabillant, lorgnée par Norman, par la caméra et donc par nous, fait naître en notre esprit l'idée de viol. Jusqu'à présent nous avons enfui nos pulsions sexuelles troubles, en leur substituant le vol, apparemment moins compromettant.

Notre inquiétude est suscitée lorsque nous apercevons l'ombre à l'autre côté du rideau. Elle ne le voit pas, mais nous savons qu'elle doit appartenir à un individu menaçant qui est désormais entré dans la salle de bain. II n'y a pas eu de son jusqu'à présent, sauf le bruissement de l'eau. Lorsque l'intrus recule rapidement le rideau de douche et commence à attaquer la femme nue avec un couteau de cuisine, des crissements forts et aigus fournissent un accompagnement étrange et sans relâche aux coups rythmiques de couteau. II semble que la violence de ce crime est issue d'un violent appétit sexuel car nous regardons l'effet de l'attaque sur la victime à l'extérieur de la baignoire, c'est-à-dire, du point de vue de l'agresseur. ${ }^{9}$

Cette scène est choquante, non pas parce qu'elle est particulièrement ensanglantée (nous ne voyons jamais le couteau entrer le corps de la femme, bien que nous l'entendons), mais parce qu'elle est inattendue, nous nous sommes entièrement identifiés avec le personnage de Marion et soudain, elle est tuée, prise par surprise quand elle était le plus vulnérable. Thomas Leitch considère que la violence de cette scène a marqué une nouvelle ère dans la cinématographie hollywoodienne. ${ }^{10}$ Aucun détail de l'acte monstrueux ne nous est épargné, les coups de couteau démentiels, la panique de la victime, les cris de douleur et de terreur.

Quand l'attaque s'arrête et le meurtrier quitte la salle de bain, elle s'éffond dans la baignoire le dos contre le mur. Son dernier acte consiste en un effort désespéré de saisir le rideau de la douche, qui est déchiré par le poids de son corps. L'eau obscure qui coule dans le drainage circulaire à la base de la baignoire est son sang. Maintenant, avec une fascination presque clinique, la caméra étudie en gros plan son visage immobile, les yeux ouverts, fixes, sans vie. La caméra se focalise sur l'un des yeux, puis se déplace lentement autour de lui dans un mouvement qui est presque mais pas tout à fait circulaire. L'appareil crée alors une fermeture de transition de la scène par le pivotement panoramique autour et en dehors de la salle de

\footnotetext{
${ }^{9}$ Jean Douchet, op. cit., p. 147.

${ }^{10}$ Thomas Leitch, 2004, p. 16.
}

bain et dans la chambre voisine. Dans une coupe qui traîne, et dont le sens est évident pour nous, la caméra regarde sur la table sur laquelle l'argent volé est caché dans un journal plié.

Dans une interview dans laquelle il affirme que " le cinéma pur est un assemblage de morceaux de film», Hitchcock donne une brève analyse de cette scène pour justifier sa conviction que "Psycho est probablement l'un des films les plus cinématographiques que j'ai jamais fait." ${ }^{11}$ Certainement beaucoup plus est en cause. Surtout dans la scène de la douche, la caméra d'Hitchcock est en fait contrôlée par des considérations qui dépassent les limites de ce qu'il appelle le cinéma pur. ${ }^{12}$ Dans plusieurs de ses écrits, il reconnaît que l'opinion publique, et ses propres préoccupations au sujet de la censure, ont impérativement demandé que les zones erogenes de la femme ne soient pas montrées à l'écran. Ainsi, la séquence artistiquement évite toute exposition sordide. Aussi, le meurtre dans la baignoire, comme Hitchcock l'avoue, est purement illusoire car le couteau ne touche jamais le corps de l'actrice, malgré la rapidité de ses coups. L'intensité de la violence et de la sexualité implicite est laissée à l'imagination des spectateurs, au but de leur provoquer des réactions affectives variées. Une des raisons pour lesquelles Hitchcock a fait le film en noir et blanc a été sa conviction que la vue du sang rouge qui coule dans l'égout aurait pu être plus que le public pourrait supporter. II a supposé, à juste titre, que l'émotion qu'il s'est efforcé de manipuler serait mieux atteinte par le montage suggestif de petits morceaux de film, plutôt que par l'utilisation sensationnelle de la couleur.

Comme l'on a déjà mentionné, la scène du meurtre se termine avec la magistrale transition de l'œil ouvert à l'argent dans le journal plié sur le bureau. Cela nous rappelle ce que la défunte avait convoité, et ainsi la prise de vue soutient notre désir de savoir ce qui va maintenant se passer avec l'argent. Hitchcock semble se moquer de nos préoccupations pour l'argent, car notre propre cupidité tout comme celle de Marion ne signifie plus rien après les violences auxquelles nous venons d'assister. La principale fonction de l'argent est désormais celle d'un «MacGuffin», terme appartenant à Hitchcock qui l'utilise pour nommer quelque chose d'arbitraire qui donne des motivations à l'action des personnages. ${ }^{13}$ Notre attention est compromise par l'inquiétude que nous sentons pour le sort de l'argent. C'est pour l'argent que nous nous préoccupons, pas pour la vie de Marion. C'est

\footnotetext{
${ }^{11}$ Irving Singer, 2004, p. 15.

12 Ibidem.

${ }^{13}$ François Truffaut, op. cit., p. 139.
} 
pourquoi le choc du meurtre est d'autant plus terrible. Notre conscience plongée dans un état d'atrophie, est maintenant terriblement secouée. Initialement horrifié et écoeuré par le meurtre, Norman regagne rapidement sa contenance pour systématiquement disposer de toutes les traces du meurtre. Voici le deuxième choc: après la perte de notre identification avec Marion, nous sommes maintenant conduis à nous identifier avec Norman car nous sommes avec lui pendant toute la scène dans laquelle il déplace le corps, le met dans le coffre de sa voiture avec tous ses biens (y compris l'argent), et pousse la voiture dans un marais. « Le « corps » (Norman) devient, à cet instant précis, notre héros, Marion (la conscience morale), comme le vol, n'en était que l'appât et l'alibi. » ${ }^{14}$ Les deux personnages reflètent notre dédoublement et ont augmenté la tension à ce que la rupture semblait inévitable. Marion, notre complice est devenue une témoigne gênante, on veut la supprimer tout comme on désirerait supprimer notre conscience morale car on voulait jouir encore de notre situation confortable de spectateur vicieux, de consommateur et de « glouton optique». 15

Douchet parle aussi de notre refuge dans notre «bonne conscience » par l'inversion de notre désir en crainte. II donne au spectateur des valences créatrices. C'est nous, dit-il, qui poussons Norman à regarder par le trou du mur, c'est nous qui lui suggérons l'idée de viol, et c'est toujours nous qui lui transmettons l'envie de tuer Marion. Le dédoublement et ainsi complet, nous sommes le protecteur et le tueur de la victime, on ressent le désir et la crainte en même temps, au summum de leur intensité. La vision de Douchet et très proche ici de celle d'Esquenazi qui affirme que la caméra hitchcokienne réussit à donner un nouveau statut au spectateur qui «n'est plus l'observateur extérieur d'une attraction, mais l'auxiliaire de l'action, ou même participant à cette action, et ceci dans un sens tout à fait concret : en effet, l'espace du récit ne devient tel que dans la mesure ou le spectateur opère, par induction, les liaisons qui reconstituent un espace plein.» ${ }^{16}$ C'est comme si le spectateur sortait de sa propre reserve, pour devenir le producteur de son propre plaisir.

Rappelons-nous le moment ou il semble que la voiture de Marion ne va pas disparaître dans le marais, mais enfin elle le fait, beaucoup au secours de Norman et à notre soulagement. Aussi, nous sommes horrifiés par son calme au cours du processus de nettoyage et par son efficacité, qui laisse à penser qu'il a dû

\footnotetext{
${ }^{14}$ Jean Douchet, op. cit., p. 146.

${ }^{15}$ Ibidem.

${ }^{16}$ Pierre Esquenazi, op. cit., p.36.
}

«nettoyer» après sa mère avant, mais aussi par le fait que nous sommes témoignes à ce nettoyage car il est le seul personnage à l'écran. Tout comme nous n'avons pas eu le choix de notre identification avec Marion, en dépit de ses imperfections, nous ne pouvons pas choisir d'éviter «être» Norman maintenant. II convient également de noter que sa dissimulation de la criminalité semble motivée par le désir du garçon de protéger sa mère, et donc il ne peut pas être totalement condamné par nous.

Les troubles éprouvés pendant cette scène nous laissent épuisés. En cet état nous regardons l'œil ouvert de Marion et les remords s'ensuivent. Hitchcock trouve ici la meilleure solution pour les affres de notre conscience : la mère criminelle. Ce coup de théâtre nous offre un coupable pour y jeter toute notre culpabilité. Ainsi le héros dont nous nous sommes identifiés jusque là n'est coupable, et nous non plus. La bonne conscience reprend sa place et Norman s'installe maintenant comme notre héros unique. A ce point nous sommes de tout cœur avec lui tout comme nous l'avons été avec Marion auparavant.

En parlant de ce tour de passe-passe, Douchet utilise les termes freudiens de ça, moi, surmoi: «Avant le crime, en effet, notre trop intense participation au spectacle a éveillé, puis activé l'obscure et redoutable puissance de nos impulsions sexuelles (le ça). Elle a ainsi provoqué l'affrontement de deux réalités constitutives de notre personnalité : le ça et le moi, qu'incarnent respectivement Norman et Marion. Sous la poussée de nos instincts, cette participation a favorisé l'écartèlement progressif, puis le clivage en deux de notre moi. Le ça s'est emparé graduellement de notre faible moi, toujours prompt à céder aux sollicitations charnelles, ainsi qu'à la douce réminiscence de la jouissance fœtale et l'a détaché complètement de ce gardien vigilant qu'est notre conscience. Son but final - et nous avons vu comment il y parvient - est de chasser, puis d'éliminer, avec la complicité inavouée de ce qu'il y a de plus sensoriel en nous, la conscience de notre moi. ${ }^{17}$ Le ça crée en nous un dédoublement imaginaire car il invente un conflit entre deux éléments de notre personnalité : le moi et le surmoi, avec la mère autoritaire et possessive, image du monde et de ses règles, en position de surmoi. Ainsi procédant, le ça tout puissant ôte à notre moi la possibilité de découvrir le vrai coupable. Le ça nous offre une image fausse de lui-même et nous pousse à fournir une image idéale de notre moi sous laquelle il puisse se camoufler.

Quand Norman reste notre seul héros, le ça reste la seule réalité psychique. Pour dissimuler son

\footnotetext{
${ }^{17}$ Jean Douchet, op. cit., p. 150.
} 
existence il prend le masque de notre bonne conscience. Ainsi, continue Douchet, «notre faible moi qui dans son affolement a besoin de se raccrocher à quelque chose, peut-il s'identifier à cet infidèle reflet qu'est ce nouvel héros qu'il s'est choisi [....] Cela lui permet de s'apitoyer sur luimême, pauvre victime d'un surmoi excessif et de s'émerveiller de sa grandeur d'âme.» ${ }^{18}$ Notre bonne conscience nous pousse à nous justifier, nous voulons et nous avons besoin de nous sentir nobles et bons.

Quand Norman précipite la voiture de Marion dans le marais, il engloutit la réalité de notre libido dans l'obscurité du subconscient. A ce moment, Norman apparaît comme la digne figure de notre moi. Des lors, remarque Douchet, dans l'état de dédoublement imaginaire dans lequel nous nous trouvons, la participation fascinée se tourne contre nous. «Fini le temps de nous jouer, comme avant le meurtre, la comédie. C'est elle, maintenant qui nous joue ${ }^{19}$

Une fois Marion au fond du marais, le film temporairement se déplace sur la forme d'un détective de théâtre plus classique, Arbogast, chargé de trouver l'argent. Celui-ce rencontre Sam et Lila (la soeur de Marion), qui sont également à la recherche de Marion. Nous sommes maintenant dirigés à nous identifier temporairement et superficiellement avec Arbogast dans sa recherche qui conduit au motel et à Norman. Comme il l'a vue assise à la fenêtre, Arbogast veut parler avec la mère de Norman, mais Norman refuse. Pour cette raison, Arbogast retourne au motel dans une nouvelle tentative de parler avec la mère. Son erreur est d'aller jusqu'à la maison, où il est poignardé à mort par la „maman”.

Et voici une autre scène familière qui dépeint l'utilisation du montage chez Hitchcock. Le détective monte prudemment les marches de l'escalier dans la maison de Bates. Nous éprouvons le suspense de son approche à la chambre dans laquelle nous savons que le danger réside. Soudain, la caméra révèle la figure menaçante de quelqu'un qui se précipite en dehors de la chambre. En gros plan on a le visage surpris du détective pendant qu'il est poignardé. Comme dans la scène de la douche, il semble que nous voyons le couteau entrer dans le corps de la victime, mais en réalité il y a seulement une série de coups simulés qui sont filmés de telle manière comme pour qu'ils paraissent consécutives. Particulièrement parce que le détective est une personne sympathique, ou du moins inoffensive, notre sentiments réactionnent

\footnotetext{
${ }^{18}$ Ibidem.

${ }^{19}$ Jean Douchet, op. cit., p. 153.
}

fortement face à ce gros plan de l' agression horrible qu'il souffre.

Quand il parle de l'agression sur le détective, Hitchcock décrit son utilisation du montage comme suit:"je me suis servi d'une seule prise d'Arbogast qui monte l'escalier et, quand il s'est approché de la dernière marche, j'ai placé la caméra en hauteur pour deux raisons: la première afin de pouvoir filmer la mère verticalement car, si je l'avais montrée de dos, j'aurais eu l'air de masquer volontairement son visage et le public se serait méfié. [..] La seconde et principale raison pour monter si haut avec la caméra était d'obtenir un fort contraste entre le plan général de l'escalier et le gros plan de sa figure lorsque le couteau s'abattait sur lui. C'était exactement de la musique, voyez-vous, la caméra en hauteur avec les violons, et soudain la grosse tête avec les cuivres. $^{20}$

Il faut remarquer ici la caméra qui est positionnée de telle manière pour soutenir le mensonge cinématographique. La scène du meurtre d'Arbogast est filmée en plongée pour qu'il ne soit pas possible de reconnaître Norman travesti en sa mère et pour exprimer le sentiment d'oppression et d'angoisse ressenti aussi bien par le personnage que par le spectateur. L'angle de prise de vue et ainsi déterminé par le conflit interne que notre dédoublement a instauré en nous. A ce moment, émotionnellement et intellectuellement traumatisés, nous sommes amenés à accepter comme incontestable l'existence de la mère.

Hitchcock sait comment choquer les téléspectateurs et il le fait avec une grande finesse. Quand on lui a suggéré que, dans Psycho, il a dirigé les spectateurs plus que les acteurs, Hitchcock a répondu: "Oui. II s'agit d'utiliser le cinéma pur pour provoquer le public à sentir." 21

La caméra d'Hitchcock soutient un état d'agitation continuelle ce que pour les critiques a fait la preuve d'un véritable cinéaste; il est, métaphoriquement parler, un « poet.» ${ }^{22}$ Hitchcock a vraiment la capacité de manipuler les sentiments du public juusqu'à tel point que nous prenons parti pour Norman contre Arbogast. Mais, on doit se rappeler que nous sommes venus voir un film d'Hitchcock pour lequel le cinéma est l'art magique des apparences,

c'est-à-dire seulement un moyen de nous duper. Nous assistons au trouble de Norman, un pauvre enfant qui vie avec une mère criminelle et nous $y$ participons, on se raccroche aux apparences cédant au goût du mélodrame. Mais le trouble de

\footnotetext{
${ }^{20}$ François Truffaut, op. cit., p. 234.

${ }^{21}$ Irving Singer, op. cit., p. 22.

${ }^{22}$ Lindsay Anderson, 2003, p. 26.
} 


\section{"Mircea cel Batran" Naval Academy Scientific Bulletin, Volume XX - 2017 - Issue 1 The journal is indexed in: PROQUEST / DOAJ / Crossref / EBSCOhost / INDEX COPERNICUS / DRJI / OAJI I JOURNAL INDEX I I2OR / SCIENCE LIBRARY INDEX / Google Scholar / Academic Keys/ ROAD Open Access I Academic Resources / Scientific Indexing Services / SCIPIO / JIFACTOR}

Norman n'est rien qu'une comédie à laquelle nous croyons. Nous commençons à désirer que la mère soit démasquée et nous sommes contre le détective qui suspecte Norman. Nous souhaitons, encouragés par notre curiosité aussi, qu'il rencontre la mère pour qu'il comprend son erreur. Et ainsi notre bonne conscience favorise encore une fois le jeu de notre «gloutonnerie optique »: «Que, nous ayant trompé sur la nature de son agent, notre libido peut en user maintenant à sa guise. Nous cédons entièrement à notre curiosité malsaine [...] Et nous voici, derechef, entrainés dans le monde vertigineux des sensations fortes, dans l'univers voluptueux du suspense viscéral. ${ }^{23}$

Quand Arbogast se lance dans la recherche de la mère, nos sensations sont tendues au maximum, divisées entre la curiosité et sa satisfaction, entre le soulagement de voir enfin la mère meurtrière et la terreur d'avoir à la regarder en face. Dans ce cas la tension ressentie est différente de celle éprouvée dans la scène du meurtre de Marion car à la différence du meurtre de Marion où les désirs et les craintes du conscient et de l'inconscient concordaient ici, ils s'inversent est s'opposent. Ici notre curiosité craint la mère, tandis que pour Marion nous n'avons éprouvé que du désir et du plaisir. Le ça se prouve encore une fois plus fort que le moi, ses désirs sont irrésistibles par rapport à ses craintes, alors que ceux du moi sont dominés, inhibés, paralysés par nos peurs. Au moment ou le détective monte les escaliers, nous craignions ce qui peut arriver pour notre monde conscient, mais la jouissance que notre inconscient manifeste pour le suspense est plus forte que notre frayeur.

Maintenant que nous avons été privés d'Arbogast comme point d'identification, nous sommes une fois de plus avec Sam et Lila. Nous cherchons refuge auprès d'eux mais ils se trouvent euxmêmes dans un état de trouble extrême. Ils vont au shérif. L'apparition de

celui-ci, habillé en robe de chambre nous calme un peu, mais il frappe un grand coup: il nous informe que la mère de Norman est morte depuis dix ans, victime d'un «meurtre-suicide», dans lequel elle et son amant ont été empoisonnés. L'anxiété et la frustration atteignent des cotes insurmontables, le mystère semble insondable. La folie s'empare de l'écran et de nous. Nous restons presque incapables de fournir de nouvelles attentes. Depuis qu'Arbogast et Sam ont vu "Mom" assise à la fenêtre, et que le public et Marion l'ont entendue et vue, nous voulons savoir qui est enterré dans le cimetière, et si, en fait, c'est la mère de Norman, qui a poignardé tous les visiteurs.

\footnotetext{
${ }^{23}$ Jean Douchet, op. cit., p. 158.
}

On doit noter que, jusqu'à l'intervention du shérif qui lui annonce qu'il est suspecté, Norman semble être installé dans une passivité confortable (la sienne, et celle du spectateur hitchcockien). II est coupé de la réalité de la même façon que le spectateur vient au spectacle pour s'évader du quotidien, oublier ses soucis, pour croire à une autre réalité. Mais ensuite, nous le voyons entrer dans la chambre de sa mère et nous entendons une conversation entre eux dans laquelle il insiste qu'elle se cache dans la cave aux fruits pendant quelques jours. Le trouble revient encore plus fort quand on croit voir Norman transporter la mère dans ses bras. La ruse d'Hitchcock pour que nous soyons persuadés de l'existence de la mère meurtrière consiste à filmer la scène avec la caméra suspendue (comme dans la scène du meurtre d'Arbogast) qui monte sans coupure, pivote, cadre tout le palier et la descente d'escalier afin de nous montrer les personnages avec un éloignement maximal et ainsi attirer notre attention sur le dialogue plutôt que sur l'action. ${ }^{24}$ Nous sommes maintenant trop influençables pour reconnaître l'artifice. Nous avons gaspillé notre énergie psychique et il nous est difficile de se rendre compte des incompatibilités de la fiction. Comment pourrait la mère avoir commis ces violences farouches dans l'état dans lequel elle semble se trouver? Pour une femme qui vient de poignarder un homme à la mort, elle semble étonnamment passive dans ses bras. D'autant plus intéressant, la scène est traitée avec assez d'objectivité pour accorder la possibilité de démasquer le mensonge. Aussi, la dispute qui s'était produite dans la chambre nous avait préparés pour le pire. Nous attendons voir un monstre, mais on ne voit qu'une vieille femme vulnérable comme un enfant. Notre attente a été trompée encore une fois. «On ne peut se moquer plus effrontément du spectateur.» ${ }^{25}$

A la fin, Norman finit par nous posséder, remarque encore Douchet. « II fait appel à ce qui reste en nous du voyeur, aux derniers soubresauts de nos sens exacerbés, aux ultimes ressources de frissons et d'extases masochistes que le spectacle n'a pas complètement épuisées. ${ }^{26}$ Nous nous ranimons quand Norman semble vouloir nous donner la clé de l'énigme. Son pouvoir sur nous est immense, on voulait voir ce qui se passe dans la chambre, mais la caméra, nous trompe encore une fois. Elle glisse par un travelling vertical lent pour arriver au plafond et effectuer une plongée similaire à celle qui nous avait illustré le meurtre d'Arbogast ce qui nous

\footnotetext{
${ }^{24}$ Lydie Decobert, 2008, p113.

25 Jean Douchet, op. cit., p. 161.

${ }^{26}$ Ibidem.
} 
rappelle le sentiment douloureux de l'angoisse éprouvée à ce moment.

Ensuite dans l'intrigue, Lila et Sam décident d'enquêter le cas de leur propre initiative et entrent dans le motel en tant que mari et femme. Lila va jusqu'à la maison pour parler à Mme Bates, comme elle le dit, "Je peux traiter avec une vieille femme malade." Bien sûr, nous savons qu'elle ne peut pas vraiment traiter avec cette «femme», ce qui génère une tension considérable qui s'accroît lorsque, pendant l'enquête de la maison, on trouve le squelette de la maman. Norman, actif cette fois-ci, habillé en femme entre avec un couteau, mais est arrêté juste à temps par Sam, qui a repris conscience. Le squelette bascule dans la chaise et la lumière jette des ombres sur le crâne aux orbites vides, créant une impression horrible que maman est toujours en vie ou que l'on doit croire aux fantômes.

En ce qui concerne la structure du film, on a déjà montré comme elle est importante pour l'évocation des sensations et des émotions fortes et pour susciter le suspense et le plaisir. Relativement à ce sujet, Bellour voit deux « récits qui glissent l'un sous l'autre » dans Psycho : «Le génie singulier de ce film est de nouer indissolublement les deux récits qui le composent en faisant de la rencontre des deux personnages le moyen de leur substitution. ${ }^{27}$

Il y a d'abord l'histoire de Marion et le vol, quand celle-ci se clôt, on a l'histoire de Norman qui pourrait commencer à l'arrivée de Marion dans l'hôtel jusqu' à la fin de l'histoire. La première histoire s'inscrit dans la seconde, tant au niveau des identifications narratives qu'à celui de la logique des instances. L'orientation du public loin de ce qui va se dérouler en réalité constitue le centre du récit et son point d'extrême attrait.

Bellour remarque aussi qu'il existe une composition ternaire parfaite dans Psycho qui vient souligner la division binaire entre les récits et les personnages: "Trois mouvements, qui se reprennent pour s'harmoniser terme à terme par des oppositions couplées, soutiennent ainsi le déploiement de la fiction et sa cohésion organique par une hiérarchisation très sûre de la répétition et de la différence. Tous trois couvrent un itinéraire qui conduit au motel; tous trois s'achèvent sur une agression meurtrière ${ }^{28}$ Le premier mouvement appartient à Marion, le

deuxième à Arbogast et le troisième à Sam et Lila.

Le postlude du film: l'explication du psychiatre qui analyse la pathologie criminelle de Norman à la station de police, a souvent été décrit comme une déception. Belour expose l'idée que cette partie

\footnotetext{
27 Raymond Bellour, op. cit., p. 295.

${ }^{28}$ Idem, p. 296.
}

contrevient non seulement au modèle classique du récit, qui demande que la fin réponde au commencement, mais au système hitchcockien lui-même: «Non pour s'y soustraire, évidemment; mais plutôt, par un supplément d'abstraction, pour en déterminer le(s) régime(s), à partir des déplacements dont le système se dote ici par rapport à lui-même en cernant au plus près les instances qui règlent son fonctionnement. ${ }^{29}$ Cette scène $a$, en partie, la fonction de donner au public quelques moments de détente après une scène très tendue, et d'expliquer certains mystères du film. Mais il sert aussi d'«excuse» aux actions de Norman en indiquant qu'il était vraiment piégé comme il l'avait pensé. Par conséquent on peut pas tout à fait le dédaigner, on lui garde si non une vague sympathie, au moins un peu de pitié.

En ce sens, le psychiatre nous dit, que c'est en fait Mme Norman Bates qui a commis les meurtres, en tant que «personnalité dominante» qui est devenu jalouse de l'attachement de Norman aux jeunes femmes qui venaient à l'hôtel. Norman est seulement sa mère maintenant, et nous ne pouvons rien lui reprocher car il n'existe plus, en fait, il avait existé seulement à moitié dès le commencement. Norman avait été vraiment né dans son piège, et il n'a jamais eu de choix à ce sujet (comme Marion peut être l'avait eu dans sa situation).

Hitchcock, toutefois, n'a pas l'intention de laisser au psychiatre le dernier mot, car cela pourrait nous laisser avec un peu trop de sympathie pour un assassin, et on pourrait oublier l'horrible nature de sa violence. C'est Mme Bates qui obtient le dernier mot, quand elle (dans la tête de Norman) suggère que Norman essaie de la blâmer pour les meurtres, et que c'est vraiment à lui la faute car elle ne peut «faire autre chose que de s'asseoir et de regarder, comme un de ses oiseaux empaillés. Quand elle conclut qu'elle «ne ferait du mal à une mouche», nous voyons une grimace malade répartie sur le visage de Norman, la plus menaçante expression que nous avons vu chez Norman. «ll nous offre dans sa cellule l'image de notre ultime reflet possible. Celui du spectateur qui s'est entièrement perdu dans les rêveries stériles du spectacle, au point de se changer luimême en pur spectacle, un spectacle dont la logique interne est coupée de la réalité et reste sans prise sur le monde des autres. ${ }^{30}$

Ce choc se terminant on se sent empêché de reposer facilement avec l'un des «explications» des assassinats, car elles ne parviennent pas à exprimer pleinement leur horreur. Nous ne pouvons ni entièrement blâmer Norman, ni

\footnotetext{
${ }^{29}$ Ibidem., p. 292.

${ }^{30}$ Jean Douchet, op. cit., p. 182.
} 
pleinement l'excuser, lui ou sa mère, parce que les deux ont contribué à créer la même situation de même que les deux ont été capturés et détruits par elle - Norman dans la personnalité, sa mère dans le corps. II serait mieux pour nous si nous pourrions blâmer quelqu'un, comme dans les films d'action, où il est habituellement assez clair qui les bons et les méchants sont, mais dans un film comme

celui-ci, nous sommes impliqués dans le mal de manière plus efficace. Douchet remarque qu'Hitchcock « pousse jusqu'à son terme logique l'attitude de base qui fut notre quand nous sommes entrés dans la salle de cinéma. Ne nous sommes-nous pas venus éprouver le grand frisson, nous réjouir des forfaits qui se commettent devant nous? Ne nous sommesnous pas livrés en pensée, sur une fille désirable, mais en détresse- et désirable parce qu'en détresse, entièrement à notre merci - à des exactions que nous n'osons accomplir dans la vie quotidienne ? Pourquoi, des lors, nous retenir de gouter à toutes les sensations, ne pas aller au bout des perversions ? $"{ }^{31}$ Nous devenons à la fois victime et assassin avec nos identifications, et nous comprenons le fait que le mal fait partie de notre propre nature. Ce mal ne peut jamais vraiment être expulsé, certainement pas en le projetant sur un «autre» qui n'est pas tout à fait nous-mêmes dans un certain sens. La morale de cette histoire n'est pas «ne soyez pas des meurtriers comme Norman, ou Marion, la cupide, car nous sommes déjà comme eux. ${ }^{32}$

Norman, notre représentation, nous dupe constamment. Selon ses propres mots lors de son dialogue avec Marion dans son bureau: «Chacun est pris dans son propre piège » Ceci est le nôtre. Le plaisir malsain, le goût des sensations fortes, le désir d'émotions fortes et violentes, les bas instincts- tout ça se paie cher car le spectateur hitchcockien s'en fait coupable dès le moment qu'il choisit de venir voir le film. Dire qu'un film comme Psycho nous comble ou nous dégoute sera loin de la vérité car si nous continuons regarder le spectacle c'est qu'il nous plaît, et s'il nous plaît nous en sommes les complices inavoués. Norman « veut nous forcer à avouer que nous avons entretenu amoureusement, dans le secret de notre être, des pensées coupables, que loin d'en avoir horreur, nous chérissons le crime ${ }^{33}$

Le plaisir que nous espérons trouver dans le spectacle de Psycho va se transformer en une épreuve de plus en plus pénible jusqu'à sa résolution par et pour notre salut. Et comment

\footnotetext{
${ }^{31}$ Ibidem., p. 181.

32 John Lyden, op. cit., p. 238.

33 Jean Douchet, op. cit., p. 181.
}

viendra le salut ? C'est ici qu'intervient la morale, s'il y existe une, peut être nous devrions reconnaître le mal en nous-mêmes et en autres, le contenir et l'exprimer en des moyens "acceptables". Une des façons qu'on peut faire cela est de se rendre aux films de ce genre, où nous recevons la catharsis de nos péchés, en participant aux actes de ceux qui sont sur l'écran plutôt que d'agir sur nos propres instincts basiques à l'extérieur du théâtre. ${ }^{34} \mathrm{Ce}$ qu'il importe de montrer, c'est, dans les rapports du spectateur et du film, la mission qu'Hitchcock attribue à ses films et au suspense qu'ils suscitent: celle de catharsis. II faut que le spectateur se «défoule» sur le plan psychanalytique, se confesse sur le plan logique, se purifie sur le plan spirituel. ${ }^{35}$

Le fait qu'Hitchcock tourne toujours le doigt acusateur à l'auditoire est une marque de son génie. II s'agit d'un principe fondamental de tous ses films de ce genre dans lequels ils nous force à regarder le mal dans la nature humaine, une nature que nous partageons tous. Certains critiques l'ont accusé de misogynie, car, apparemment, ses femmes ont tendance à se blâmer ou à se punir pour s'être affirmées sexuellement; on pourrait penser à la mère de Norman, ou à Marion, qui a tenté se faire valoir par le biais de son vol, et qui est représentée sexuellement pendant sa douche et dans la première scène avec Sam. Mais Hitchcock ne veut pas dire que Marion mérite de mourir, parce que le public s'identifie avec elle, et lui, il comprend qu'elle avait juste essayé de sortir de son propre piège.

Des interprétations psychologiques ont aussi été attachées aux analyses féministes en suggérant que l'angoisse de castration des hommes est à l'origine de la nécessité de voir les femmes détruites, tandis que les femmes spectateurs de ces films, elles ne peuvent être pitiées que comme des masochistes qui se font complices de leur propre destruction. Mais pas tous les féministes ont accepté ce point de vue, précisément parce que certains d'entre eux aiment les films d'horreur et ne pensent pas que de tels films soient misogynes, comme l'on croit souvent. ${ }^{36}$ Par exemple, Cherry Brigid a démontré que les études montrent que les femmes reçoivent du plaisir à regarder certains de ces films pour les mêmes raisons que les hommes. Notamment, elles sont capables d'exprimer et de mieux maîtriser leurs craintes. En fait, certaines études suggérent que les femmes

\footnotetext{
${ }^{34}$ John Lyden, op. cit., p. 227.

${ }^{35}$ Jean Douchet, op. cit., p. 186.

36 John Lyden, op. cit., p. 227.
} 


\section{"Mircea cel Batran" Naval Academy Scientific Bulletin, Volume XX - 2017 - Issue 1 The journal is indexed in: PROQUEST / DOAJ / Crossref / EBSCOhost / INDEX COPERNICUS / DRJI / OAJI I JOURNAL INDEX I I2OR / SCIENCE LIBRARY INDEX / Google Scholar / Academic Keys/ ROAD Open Access I Academic Resources / Scientific Indexing Services / SCIPIO / JIFACTOR}

font semblant d'avoir peur, parce qu'on s'attend à cela d'elles. ${ }^{37}$

Qu'on soit des hommes ou des femmes, on ne peut pas projeter la faute sur l'un ou l'autre de ses personnages sans reconnaître notre propre complicité aux crimes. II semblerait que les personages sont «sacrifiés pour nos péchés», ce qui signifie que nous participons à leurs souffrances tout comme ils participent à la nôtre, car leurs péchés sont les nôtres aussi. Nous ne pouvons pas simplement transférer de la culpabilité à un «bouc émissaire», sans sentir une connexion avec ce sentiment de culpabilité. Ce fait, Hitchcock l'a rendu plus clair que personne. Néanmoins, certains réalisateurs n'ont pas reussi à rendre ce lien aussi clair, essayant au contraire de projeter la faute sur quelqu'un d'autre sans offrir aux spectateurs la possibilité de prendre la responsabilité. Les plus efficaces des drames, observe John Lyden, ne le feront pas. ${ }^{38}$ Oedipus Rex, par exemple, est une grande tragédie justement parce qu'elle nous implique dans le mal et fait que nous éprouvons aussi bien de l'horreur envers le crime, ainsi que de la sympathie pour les criminels.

Même s'il ne l'admet, Hitchcock a joué avec le thème oedipal et les côtés moralisateurs de la vie tout au long de ses films. Eric Rohmer et Claude Chabrol ont observé le fait que tous les films de Hitchcok de The Lodger (1926) à Psycho(1960), ont des implications qui dépassent la situation critique des personnages eux-mêmes. ${ }^{39}$ Mais il l'a fait avec un formidable sens de l'humour, comme si tout n'était qu'une grande blague privée à laquelle le public pourrait participer. Psycho, en particulier, ne devrait pas être réduit à un message moral sérieux, car il s'agit d'un film d'Hitchcock bien regardé comme un morceau de «divertissement» pour un public qui se laisse conduire d'un personnage à l'autre sans savoir où il s'arrêtera. On voit ici que la comparaison avec la montagne russe fonctionne très bien car le spectateur jouit de cette désorientation cinématographique de la même manière que les gens qui aiment les manèges des parcs d'attractions, à la fois excitantes et effrayantes. L'élément clé est que le trajet se termine, pour que nous puissions réaliser qu'il s'agissait seulement d'un «jeu», et c'est là que réside le plaisir de l' expérience.

Thomas Leitch a dit que les films d'Hitchcock sont mieux compris comme une sorte de jeu où les choses qui, normalement, causent des douleurs sont maîtrisées par le biais de l'humour

\footnotetext{
${ }^{37}$ Brigid Cherry, Refusing to Refuse to Look, cité dans Lyden, op. cit., pp. 227- 228.

${ }^{38}$ John Lyden, op. cit., p. 240.

${ }^{39}$ Claude Chabrol, 2006, p. 116.
}

et la capacité de nous «réveiller» du cauchemar après. Nous pouvons nous laisser aller et rouler avec les personnages par l'intermédiaire du divertissement, et ainsi le renforcement à l'extérieur de la norme, nous permettra de mieux faire face à la vie quotidienne lorsque nous y retournons. ${ }^{40}$ Cet avis se rapporte à l'idée que le cinéma offre une expérience qui permet l'entrée dans un monde imaginaire afin d'assumer des rôles interdits pour que nous puissions revenir au monde réel renouvelés. Le fait que les films d'Hitchcock offrent du plaisir ludique, toutefois, ne signifie pas qu'ils n'ont pas des observations morales sérieuses à faire. Ils prouvent ce qu'il veulent sous la forme du jeu, mais cela ne diminue pas l'importance de ces observations. Les jeux sont des choses très sérieuses dans le sens que, tout comme l'humour, ils nous permettent de traiter avec ce qui est trop effrayant pour être directement confronté. Dans Psycho, Hitchcock nous permet de sonder le mal de nos propres pulsions, et d'y réfléchir pour obtenir la libération de leur catharsis, tout comme dans la tragédie grecque. 40 Thomas M. Leitch, 1991, p. 260-261, cité par Lyden,
op. cit., p. 240.

(c) 2017. This work is licensed under the Creative Commons Attribution-Noncommercial-Share Alike 4.0 License. 


\section{"Mircea cel Batran" Naval Academy Scientific Bulletin, Volume XX - 2017 - Issue 1 The journal is indexed in: PROQUEST / DOAJ / Crossref / EBSCOhost / INDEX COPERNICUS / DRJI / OAJI I JOURNAL INDEX I I2OR / SCIENCE LIBRARY INDEX / Google Scholar / Academic Keys/ ROAD Open Access I Academic Resources / Scientific Indexing Services / SCIPIO / JIFACTOR}

\section{Conclusions}

A la base de cet article se trouve la conviction qu'il est possible de comprendre l'attrait du cinéma comme un formidable révélateur de plaisir. Effectivement, on a démontré comment les films peuvent susciter du plaisir pour le spectateur. Pour y arriver, nous avons analysé Psycho, une oeuvre qui défie les règles et perturbe le spectateur dans un mélange étrange de plaisir et de trouble. Nous sommes partis de la prémisse qu'un bon film provoque du plaisir. C'est pour cela que nous avons choisi Psycho comme objet d'analyse. II est un des plus fameux films de l'histoire cinématographique donc un des plus efficaces convoyeurs de plaisir. C'est évident. Sinon pourquoi les gens auraient payé pour aller le voir ? Dans Psycho, Hitchcock semble avoir une compréhension profonde de la nature du «plaisir convenable » car il sait comment accroître la participation et le suspense. II sait comment faire le spectateur s'identifier avec les personnages, comment bien structurer le film pour que, avec chaque scène et avec chaque séquence, le suspense soit plus élevé que dans la précédente jusqu'à l'apogée de l'émotion et du plaisir dramatique. Avec Psycho, nous devenons à la fois victime et assassin avec nos identifications, et nous comprenons le fait que le mal fait partie de notre propre nature. Le plaisir malsain, le goût des sensations fortes, le désir d'émotions fortes et violentes, les bas instincts- tout ça se paie cher car le spectateur hitchcockien s'en fait le complice inavoué, coupable dès le moment qu'il choisit de venir voir le film. C'est ici qu'intervient la morale, se rendre aux films de ce genre, pour recevoir la catharsis de nos péchés, en participant aux actes de ceux qui sont sur l'écran plutôt que d'agir sur nos propres instincts basiques à l'extérieur du théâtre.

\section{Bibliographie}

[1] ANDERSON, Lindsay. Sequence and Rise of Auteurism in 1950's Britain, dans British Cinéma of the 1950's: A Celebration, édité par lan Mackillop et Neil Sinyard, Manchester University Press, 2003.

[2] BELLOUR, Raymond. L'analyse du film, Ed. Albatros, Paris, 1979.

[3] CHABROL, Claude, ROHMER, Eric. Hitchcock, Editions Ramsay, 2006.

[4] DECOBERT, Lydie. L'Escalier dans le cinéma d'Alfred Hitchcock. Une dynamique de l'effroi, Ed.

L'Harmattan, Paris, 2008.

[5] DOUCHET, Jean, Cahiers du Cinéma, 1999.

[6] ESQUENAZi, Jean Pierre. Hitchcock et l'aventure de Vertigo. L'invention à Hollywood, CNRS Editions, Paris, 2001.

[7] HOBERMAN, J. Voice Film Guide. 50 Years of Movies from Classics to Cult Hits, John Wiley \& Sons, Inc, New Jersey, 2007.

[8] LEITCH, Thomas. Crime Films, Cambridge University Press, 2004.

[9] LEITCH, Thomas. Find the Director, and Other Hitchcock Games, University of Georgia Press, 1991.

[10] LYDEN, John C. Myths, Morals, and Rituals. Film as religion, New York University Press, 2003.

[11] SINGER, Irving. Three Philosophical Filmmakers Hitchcock, Welles, Renoir, the MIT Press, Cambridge, Massachusetts, 2004

[12] STERRITT,David, The Films of Alfred Hitchcock,Cambridge University Press, 1993.

[13] TRUFFAUT, François, Hitchcock, Editions Ramsay, 1983. 